\title{
SMART CROWD MANAGEMENT USING LOCATION BASED SERVICE
}

\author{
A.Sharmila Agnal \\ Assistant Professor \\ Department of Computer Science, \\ SRM Institute of Science and Technology \\ Chennai, Tamil Nadu
}

\begin{abstract}
Advancement of mobile communication technology and popularization of information technology, diversified value-added services have emerged. Location-Based Services (LBS) is an application of information and Internet integrated technology that has been developed rapidly in recent years. This study utilized a room-based indoor location mode to construct a wireless sensor network location-oriented service platform. This platform could meet the indoor location search by combining the WIFI wireless location technology. Based on the concept of LBS, the indoor locationoriented service could be realized by combining the WIFI location technology and room-based location mode, in order to achieve a ubiquitous campus environment. Users only need to utilize WIFI Tagged with PDA through the cloud, and a wireless network to $\log$ in this information platform, so as to receive the services of the ubiquitous campus. The preliminary study has completed this information platform and implemented it in this campus environment with satisfactory results.
\end{abstract}

Key Words:- Human Computation, LocationBased Services, Crowdsourcing,

\section{INTRODUCTION}

This paper proposes a location privacy protection method based on differential privacy strategy based on big data in sensor networks. This method expresses the position data set by constructing the location information tree model, this solves the problem that location data is difficult to be expressed because of its characteristics of high depression and low density and add noise expression to cover the noise trajectory and original position data. It is more effective in protecting the privacy of data and maintains high availability of data and algorithms. Previous forms of this LBS (Location Based Services) has never been used for any such indoor application and present systems only show

\author{
Arnav Doley, Kaushik Iswary, Sagar Soni \\ Final Year Students \\ Department of Computer Science, \\ SRM Institute of Science and Technology \\ Chennai, Tamil Nadu
}

the reviews and the location of the particular place and never the density of people in a particular building nor does it take into account the condition that the state of the building is in which is very accountable for accessing the situation in an extremely crowded place. Our application tends to take all this data from modular sources like the temperature sensor and the humidity sensor then the live camera feed from a certain location in order to access the situation in that place and will be able to take the necessary steps in order to disperse the crowd or to distribute the people throughout the particular location and this is the use and the practical application of the application that we are going to build. The figure number one depicts the flow of work through which our application is going to work.

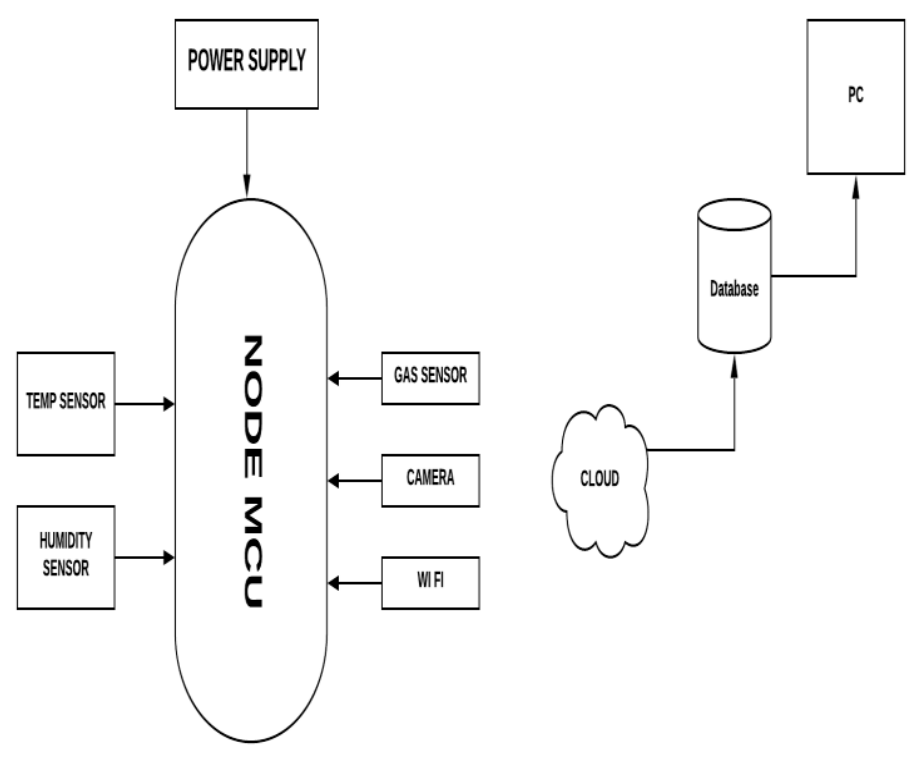

Fig. 1: Flow Of The Application 


\section{PROBLEM STATEMENT}

Location-Based Services have been presented throughout the present generation. If we look at the likes of UberRush and other such service providers then we can have a look at how their application works, It provides a person that is going from point $\mathrm{A}$ to point B certain task are which in the case of the UberRush is delivering a certain good and this task is presented for a person who is going to that particular location. This is what is known as a crowdsourcing service where a person who is on his way from one point to another is able to get the most benefits out of his journey to those points.

So the problem that we are looking to find a solution for is that any place or any such crowdsourcing services provided only the location and the areas through which a person is traveling to and gaining the most out of his/her journey but in our case we are going to use a modified version of this technique to be able to provide the users of our application the ability to look at the current situation in particular building and then redirecting those people to a place that is less crowded as well as providing them with the best experience for those people as well as controlling the crown in a certain place. This is not only beneficial to all the businesses that are established nearby but also helps to prevent crowding in a particular area and our model also tracks the current condition in a building so that a person can decide whether they want to be there or not.

\section{SYSTEM ARCHITECTURE}

The application that we are designing os going to consists of an IoT Based Location Searching System along with the use of sensors which consists of Temperature and Humidity sensor. We are going to use a NodeMCU as an Analog to Digital Converter(ADC) which is going to convert the signals from an analog signal to a digital signal. From all this we are going to decide a threshold value and the optimum temperature required to sustain a comfortable environment can be calculated from that threshold value. Also each of the building are required to have a static camera fixed on each of its floors which is true for any business and these camera values are then send from the nodeMCU to the server where that video can be analyzed and the density if the crowd can be known in that particular place.

\subsection{MODULES}

\subsubsection{CROWD ANALYSIS}

- Pre Processing: Reading the images that are made available to the server into a data format that can be used for selective background modeling.

- Selective Background Modelling: Using a background subtraction algorithm we are going to create a mask so that we are able to differentiate between what is the background as well as what is the objects that are not a part of the background which in our case will be the people moving around the floors.

- Background and Foreground Detection: As already stated above we are going to separate the foreground from the background after the selective background modeling has been conquered. This method will classify the selective background and necessary background and foreground objects by identifying its pixel from the input image.

- Data Validation: During this stage we are going to examine the binary mask of the background and foreground object with the input image and then background so that we are able to provide the necessary data to calculate the density of people on that particular floor.

The figure number 2 depicts the way the this particular module is going to work as well as give the flow of direction of the process through which it is going to work.

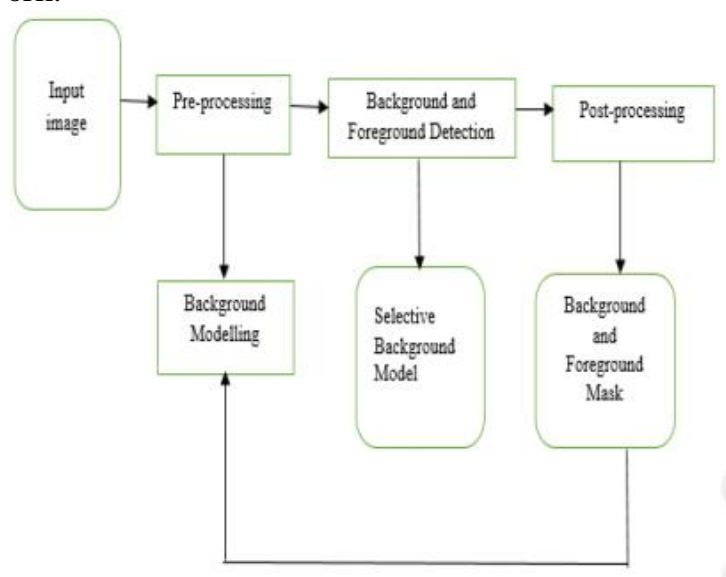

Fig. 2: Crowd Analysis Module

\subsubsection{TEMPERATURE AND HUMIDITY SENSOR}


- Humidity and Temperature sensors are going to be placed on each of the floors along with the cameras which are going to measure the ambient temperature as well as the humidity of all the floors in the building in order to create a threshold of the condition of the particular building and upload its state to our servers.

- The NodeMCU is going to send a signal to the DHT module which is turn is going to return a response signal containing the temperature as well as the humidity data. As soon as this data is received the collected data is then extracted into two parts one containing the humidity readings and the other containing the temperature reading and through the nodeMCU this data is then sent live to the servers for the final computation.

- The sensor that has been used for this module if the DHT11 and this module features a humidity and temperature complex with a calibrated digital signal output meaning that the DHT11 is a combined module for sensing both the temperature as well as the humidity of the surrounding and it gives a calibrated digital output signal. It gives us precise values of humidity and temperature and ensures high reliability and long term stability. This sensor has a resistive type humidity measurement component and NTC type temperature measurement component with an 8-bit microcontroller inbuilt which helps to make the repose time of this module that much faster and this is perfect for measuring the data in real-time which our project uses.

\subsection{ALGORITHMS}

\subsubsection{BACKGROUND SUBTRACTION}

Background Subtraction is a method through which the foreground and the background of an image or a static video can be differentiated upon meaning that we are able to extract the background which is a static object and the remaining objects in the background which are the variables can be extracted and then we are able to count out those variables and find out the density of people in a particular floor and subsequently the whole building.

- The Problems: There are a few obstacles that we come through with this algorithm. First of all the video which in our case will be coming from the cameras installed on each of the floors needs to be static in order for this algorithm to work properly. We also have to handle the static background geometry like the tables chairs cupboards and have to make sure that these things remain static in order to get an accurate value or reading,

The Basic Model: The basic of our module consists of the frame differencing technique where it subtracts one frame from the other.

|frame(i)-frames(i-1)|>Th

While maintaining Th(threshold value) and this algorithm is very sensitive to the threshold value.

Frame Differencing: Background is estimated to be the previous frame and we can show this frame differencing as follows

$$
\begin{aligned}
& B(\mathbf{x}, \mathbf{y}, \mathbf{t})=\mathbf{I}(\mathbf{x}, \mathbf{y}, \mathbf{t}-\mathbf{i}) \\
=>\mid I(x, y, t) & \mathbf{I}(\mathbf{x}, \mathbf{y}, \mathbf{t}-1) \mid>\mathbf{T h}
\end{aligned}
$$

Depending on the object structure the frame rate and the global threshold this approach may or may not be useful but in our case of application is the perfect solution to be able to count the number of people on a particular floor of the building.

In the case that the background is more likely to appear ina particular frame then we can use the median of the previous (n) frames as the background module and then we get the following

$\mathbf{B}(\mathbf{x}, \mathbf{y}, \mathbf{t})=\operatorname{median}\{\mathbf{I}(\mathbf{x}, \mathbf{y}, \mathbf{t}-\mathbf{i})\}$

$=>\mid \mathbf{I}(\mathbf{x}, \mathbf{y}, \mathbf{t})$-median $\{\mathbf{I}(\mathbf{x}, \mathbf{y}, \mathbf{t}-\mathbf{i})\} \mid>\mathbf{T h}$

Where I belongs to $\{0,1,2, \ldots . . . . ., n-1\}$

\subsubsection{ROUTE RECOMMENDATION}

\section{THE COMPLETE PROCESS}

\subsection{AN OVERVIEW}

Our application will have two users one being the general public and then the admins who are the executives of those buildings who are able to control the modules that have been placed over at the building.

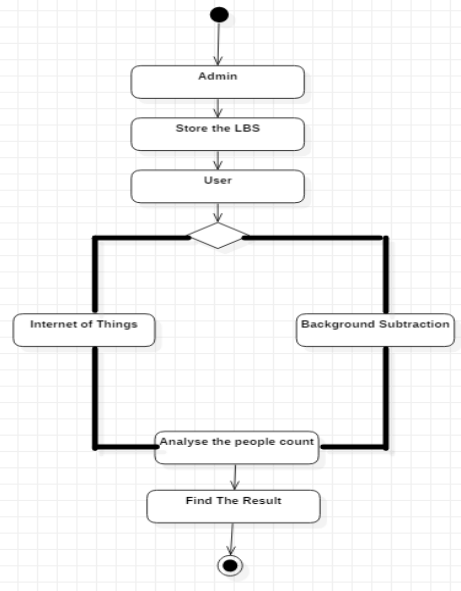

Fig. 3: Activity Diagram 
In the above diagram we are able to see how this is going to function where the admin installs the modules onto each floor of the building and the data starts accumulating at the server which is then using IoT and the aforementioned algorithms are able to analyze the people count and then are able to maintain also find out the current condition of the building then lead to the release of the final result.

\subsection{USER CASES}

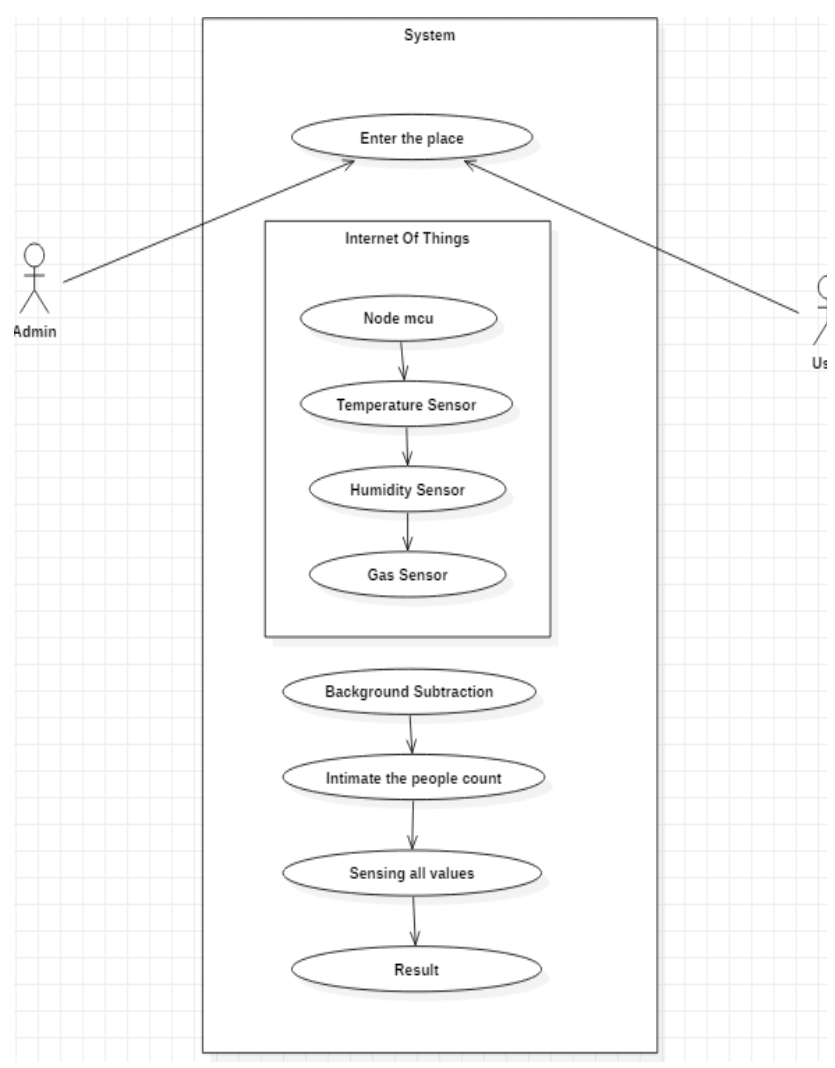

Fig. 4: User cases for the module

Diagram number four shows us how the system is going to when both the users as well as the admin are logged into our application service. An important thing to remember here is that all the computation will not occur at the location and will be done on a back endless server where all the logic of the algorithm will be put into place and the modules placed in the building will only feed the live data onto those servers where all the computation occurs and also this is used as profile of the IoT service in order to deploy the necessary functions. Also each of the users is required to be logged into a mobile application of our design so that they are able to see the results and the final route plan for their destination.

\subsection{DATABASE HANDLING}

All the data that we are going to receive from the various sensors, as well as the live feed from the camera, is going to be given to our backend database first and in our case we will be using the readily available Firebase backend software from google.

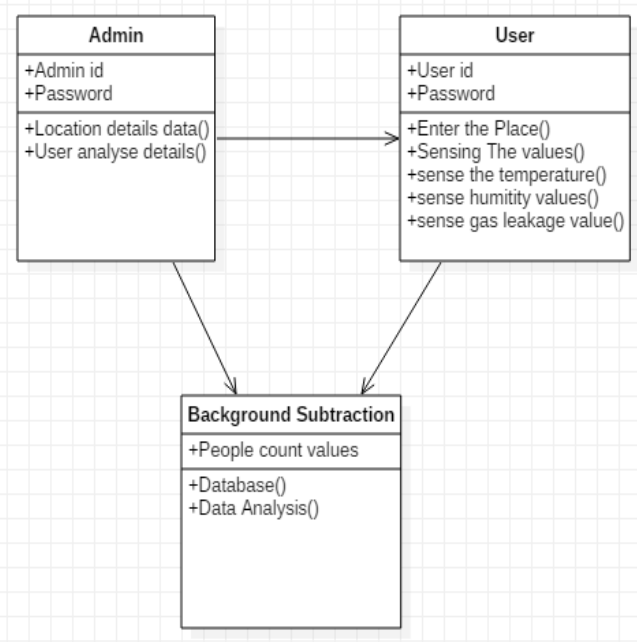

Fig. 5: Database Module

As can be seen in figure number five there are three different ways the data are going to be moduled in the backend service. The Admin side is going to contain the values received from the various sensors as well as the live feed from the camera are going to be tagged in with the admin id which in turn is going to be the id of the entire building and we are doing this so as to keep the data traffic short on the side of the backend. Then we are going to have the user side of the data modeling which will consist of the location or the place that they want to go to and then after the computation the results will be placed onto their apps which will be the route recommender for the best experience. And finally, we have the use of the background subtraction module which will be responsible for getting the count of the number of people that are going to come into the display of the frame as well as creating frame enumerations for finding the optimal solution to the live feed video that is being received from the building. As can be seen in the above-mentioned diagram that the database analysis and the people count are being fed into our database. 


\subsection{INTERACTIONS}

The order in which the application is going to work can be seen in the given figure number six.

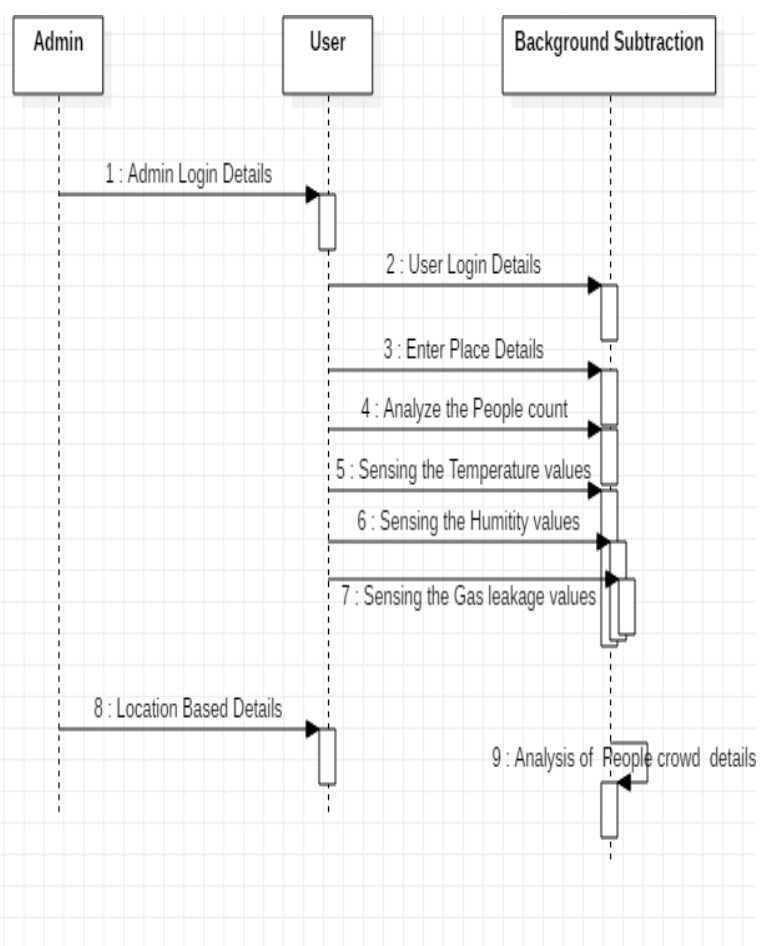

Fig. 6: Sequence model

In the following sequence model we are able to see how the whole procedure is going to work throughout the program and the levels at which they are going to operate. Another of the usage patterns that can be noticed from the diagram is the way these values are being placed one at a time towards the user as the use is going to take all the data from the admin side make the analysis then once the result has been found out then take this process to the backend server where all the computation is going to occur and then finally we are able to get the location recommender to work through and the final results are then sent onto the user. All this together are going to come together and then we will be able to make the application work for the users to be able to get the best experience as well as control the crowds with precision from one building to another depending on which of the building are attached or has applied to our program.

\section{CONCLUSION}

This paper proposes a location privacy protection method based on differential privacy strategy based on big data in sensor networks. This method expresses the position data set by constructing the location information tree model, this solves the problem that location data is difficult to be expressed, because of its characteristics of high depression and low density and add noise expression to cover the noise trajectory and original position data. It is more effective in protecting the privacy of data and maintains high availability of data and algorithms.

\section{ACKNOWLEDGEMENT}

The completion of this undertaking would not have been possible without the participation and assistance of my project team members Mr.Arnav Doley, Mr.Sagar Soni and Mr.Kaushik Iswary along with endless support from faculty members of my institution and $i$ would like to thank each and every one of them.

\section{REFERENCES}

1. Bohm, B. Murtz, C. Sommer, and M. Wermuth, (13-15 Sept. 2005,) "Locationbased ticketing in public transport", Proceedings of Intelligent Transportation Systems,pp. 194- 197.

2. Maglogiannis, and S. Hadjiefthymiades, (Vol. 76, Issue 10, October2007,) "EmerLoc: Location-based services for emergency medical incidents", International Journal of Medical Informatics,pp. 747-759

3. S. Ahmad, R. Eskicioglu, and P. Graham, "Design and Implementation of a Sensor Network Based Location Determination Service for use in Home Networks", Mobile Adhoc and Sensor Systems (MASS), 2006 IEEE International Conference on, Oct. 2006, pp. $622-626$.

4. Sadoun, and O. Al-Bayari,(3 November 2007, Vol. 30, Issue 16), "Location based services using geographical information systems", Computer Communications, pp. 3154-3160.

5. Z. Ting, X. Zhang, and O. yuanxin,(2006 2nd IEEE/IFIP International Conference in Central Asia on Internet, Sept. 2006,) ,“A Framework of Networked RFID System Supporting Location Tracking",pp.1-4.

6. M. Jakob, M. Grobmann, N. Honle, and D. Nicklas, "DCbot:Exploring the Web as Value-Added Service for Location-Based Applications", The 21st International Conference on Data Engineering 
(ICDE),Tokyo, April 5- 8, 2005.

7. C. Xiuwan, Z. Feizhou, S. Min, and L. Yuanhua, "System Architecture of LBS Based on Spatial Imformation Integration", International Geoscience and Remote Sensing Symposium (IGARSS), 2004, Vol. 4, pp. 2409- 2411.

8. C. T. Wu, and H. Mei, "Location-Based Services Roaming Based on Web Services", Proceedings of the $19^{\text {th }}$ International Conference on Advanced Information Networking and Applications (AINA), 2005, Vol. 2, pp. 277- 280.

9. J. P. Munson, and V. K. Gupta, (Proceedings of the 2nd International Conference on Mobile Commerce, 2002),"Location-Based Notification as a General-Purpose Services", pp. 40- 44 . 